\section{FRI0165 MICRORNAS DYSREGULATION IN MONOCYTES AND T CD4 LYMPHOCYTES FROM PATIENTS WITH AXIAL SPONDYLOARTHRITIS}

O. Fogel ${ }^{1}$, A. Bugge Tingaard ${ }^{2}$, M. Fagny ${ }^{1}$, N. Sigrist ${ }^{1}$, E. Roché ${ }^{1}$, L. Leclère ${ }^{1}$, J.F. Deleuze ${ }^{1}$, M. Dougados ${ }^{3}$, C. Miceli-Richard ${ }^{3,4}$, J. Tost $^{1} .{ }^{1}$ Laboratory for Epigenetics and Environment, Centre National de Recherche en Génomique Humaine, Institut François Jacob, CEA, Evry, France; ${ }^{2}$ Biomedicine, Aarhus University, Aarhus, Denmark; ${ }^{3}$ Rheumatology, Cochin Hospital; ${ }^{4}$ Immunoregulation unit, Department of Immunology, Pasteur Institute, Paris, France

Background: MicroRNAs have been shown to play a crucial role during innate or adaptive immune response. Dysregulation of miRNAs has been described in several autoimmune or rheumatic diseases including rheumatoid arthritis, inflammatory bowel disease or psoriasis. In spondyloarthritis $(\mathrm{SpA})$, only few studies on miR expression have been reported with highly diverse methodologies and involving small samples of patients.

Objectives: Because T CD4 lymphocytes and monocytes are important cells in SpA pathophysiology, we wanted to assess the miR expression profile in these two cell types sorted from axial SpA (AxSpA) patients.

Methods: Eighty one AxSpA patients were included in this study. Among these patients, 74 fulfilled the ASAS classification criteria (imaging arm) with sacro-ilitis on X-rays $(n=56)$ or objective signs of inflammation on MRI $(n=18)$. Two independent cohorts of 22 and 59 SpA patients were compared to 17 and 38 age and sexmatched controls. Both SpA patients and controls were recruited from October 2014 to July 2017 in the department of rheumatology at Cochin Hospital in Paris, France. All SpA patients had an active disease despite NSAIDs intake (mean BASDAI score of $49 \pm 19$ and mean ASDAS score of $3 \pm 0.9$ ), were free of any biologic treatment and were eligible for a TNF-blocker treatment. Seventy-seven percent were HLA-B27 positive. T lymphocytes and monocytes were isolated from PBMC by direct isolation with magnetic microbeads (CD4 +and CD14+). Threehundred seventy two miRs were screened by q-RT-PCR on the exploratory cohort and only MiRs showing a significant differential expression in the first cohort were analysed in the validation cohort. An unpaired T-test was used for comparison of miR expression level.

Results: In the exploratory cohort, 51 (CD14+) and 70 miRs (CD4+) were found to be differentially expressed between patients and controls. Among these, 15 miRs (in CD14+), and $12 \mathrm{miRs}$ (in CD4+) were also found dysregulated in the validation cohort. These validated miRNAs were found to play a key role in physiological pathways such as NFkB or TGFb, Wnt signalling and monocyte differentiation that have been involved in the pathophysiology of the disease. Neither clinical subphenotypes nor biological parameters were associated with different profiles of miR expression after adjusting for multiple tests. We found a negative correlation between miR-146a-5p level and BASDAl $(r=-0.28, p=0.011)$ and ASDAS $(r=-$ $0.38, p=5.910-4)$ in monocytes.

Conclusions: We found a dysregulation of miR expression in monocytes and $T$ CD4 lymphocytes from patients with axial spondyloarthritis, whose consequences could contribute to the pathophysiology of the disease and be of interest for therapeutic perspective. Moreover, identifying biomarkers with the potential of diagnostic signature should help the clinician in daily practice.

Disclosure of Interest: None declared

DOI: 10.1136/annrheumdis-2018-eular.7073

\section{FRI0168 PHENOTYPIC, FUNCTIONAL AND MOLECULAR CHARACTERISATION OF IL-17+CD8+ T CELLS IN HUMAN HEALTH AND PSORIATIC ARTHRITIS}

U. Srenathan ${ }^{1}$, K.J. Steel ${ }^{1}$, M.L. Ridley ${ }^{1}$, B.W. Kirkham ${ }^{2}$, L.S. Taams ${ }^{1} .{ }^{1}$ Centre for Inflammation Biology and Cancer Immunology, Dept Inflammation Biology, King's College London; ' Department Rheumatology, Guy's and St Thomas' Hospital, London, UK

Background: Psoriatic arthritis (PsA) is a spondyloarthritis affecting the joint and skin. The genetic association with HLA Class I and the clinical efficacy of IL-17A inhibitors in the treatment of PsA suggest a potential role for IL-17+CD8+T cells in PsA pathogenesis. This concept is supported by our previous data showing that $\mathrm{IL}-17+\mathrm{CD} 8+\mathrm{T}$ cell frequencies are increased in the synovial fluid (SF) vs the peripheral blood (PB) of patients with PsA ${ }^{1}$. We hypothesise that IL-17 +CD8+T cells are pro-inflammatory contributors to PsA pathogenesis.

Objectives: To perform molecular, phenotypic and functional characterisation of IL-17+CD8+T cells.

Methods: Healthy donor PB CD8 +T cells were cultured for 3 days with anti-CD3/ CD28 mAb, IL-1 $\beta$ and IL-23 to induce IL-17 +CD8+T cells in vitro, whilst PB and $\mathrm{SF}$ mononuclear cells were isolated from patients with PsA and stimulated ex vivo with PMA/ionomycin. Cytokine secretion assays were used to sort IL-17+CD8+T cells, from which RNA was extracted for RNA-sequencing of PSA PB and SF cytokine-secreting T cells. 24 hour supernatants were generated from cultured sorted cells for cytokine analysis (Luminex) or culture with fibroblasts (IL-6 and IL-8 secretion measured by ELISA).
Results : In vitro-generated IL-17 +CD8+T cells produced significant levels of IL17A, IL-17F, IFN- $\gamma$, TNF- $\alpha$, IL-22 and GM-CSF (10-9000 pg/ml range), but little $\mathrm{IL}-10$. Flow cytometry showed that in vitro-generated IL-17 +CD8+T cells coexpressed IFN- $\gamma$ (median $80 \%)$, TNF- $\alpha(40 \%)$ and GM-CSF (35\%) at comparable frequencies to ex vivo PsA synovial IL- $17+$ CD $8+$ T cells $(70 \%, 50 \%, 55 \%$ respectively). Whilst only $5 \%$ of PsA SF IL-17+CD8+T cells co-expressed the MAIT cell marker $\mathrm{V} \alpha 7.2,50 \%$ of in vitro-generated IL-17 +CD8+T cells co-expressed $V \alpha 7.2$. The cytokine profile of in vitro-generated $V \alpha 7.2+$ and $V \alpha$ 7.2- IL-17 +CD8+T cell supernatants was however comparable, sharing the cytokine profile of total IL-17 +CD8+T cells. Functionally, in vitro-generated sorted IL-17 +CD8+T cell culture supernatants enhanced IL- 6 and IL- 8 production by synovial tissue fibroblasts from patients with PsA compared to IL-17- counterparts, thus exhibiting proinflammatory capacity; we will also determine if this response is IL-17 and/or TNFmediated. Additionally, the majority of synovial IL-17+CD8+T cells co-expressed cytotoxic molecule Granzyme B, which may contribute to PsA pathogenesis. Finally, RNA-sequencing revealed that IL-17+CD8+T cells from PsA synovial fluid displayed a distinct transcriptomic signature compared to PB IL-17 +CD8+T cells as well as to synovial Th17 or Tc1 cells.

Conclusions : In vitro-generated and ex vivo-derived synovial IL-17 +CD8+T cells display a type 17 profile, as evidenced by flow cytometry and Luminex. In contrast to ex vivo PsA synovial IL-17+CD8+T cells, $50 \%$ of in vitro IL-17 +CD8 $+T$ cells co-express $V \alpha 7.2$; however, both $V \alpha 7.2+$ and $V \alpha 7.2$ - subsets share a similar cytokine profile. Functionally, IL-17+CD8+T cells exhibit pro-inflammatory potential, upregulating IL-6 and IL-8 production via fibroblasts. Analysis of our RNA-sequencing data will further reveal the molecular profile of human IL-17 +CD8+T cells, and how they may contribute to joint inflammation in PsA.

\section{REFERENCE:}

[1] Menon, et al. Arthritis Rheum 2014;66(5):1272-1281.

Acknowledgements: Funded by King's Health Schools (MRC DTP), King's Health Partners R and D challenge award, Novartis and NIHR BRC.

Disclosure of Interest: U. Srenathan: None declared, K. Steel: None declared, M. Ridley: None declared, B. Kirkham Grant/research support from: Abbvie, Novartis, Roche, UCB, Speakers bureau: Eli Lilly and Co, Janssen, Novartis, L. Taams Grant/research support from: UCB, Novartis, GSK and Novo Nordisk A/S Speakers bureau: UCB, Novartis

DOI: 10.1136/annrheumdis-2018-eular.5242

FRIDAY, 15 JUNE 2018

Spondyloarthritis - clinical aspects (other than treatment)

\section{FRI0169 FIRST VALIDATION OF CONSENSUS DEFINITIONS FOR MRI LESIONS IN THE SACROILIAC JOINT BY THE ASSESSMENTS IN SPONDYLOARTHRITIS INTERNATIONAL SOCIETY (ASAS) MRI GROUP}

W.P. Maksymowych ${ }^{1}$, U. Weber ${ }^{2}$, S.J. Pedersen ${ }^{3}$, X. Baraliakos ${ }^{4}$, P. Machado ${ }^{5}$, J. Sieper ${ }^{6}$, D. Poddubnyy ${ }^{6}$, S. Wichuk ${ }^{1}$, R.G. Lambert ${ }^{7}$, M. Østergaard ${ }^{8} .{ }^{1}$ Medicine, University of Alberta, Edmonton, Canada; ${ }^{2}$ Rheumatology, King Christian 10th Hospital for Rheumatic Diseases, Graasten; ${ }^{3}$ Rheumatology, Gentofte Hospital, Copenhagen, Denmark; ${ }^{4}$ Rheumatology, Rheumazentrum Ruhrgebiet, Herne, Germany, ${ }^{5}$ Rheumatology, University College London, London, UK;

${ }^{6}$ Rheumatology, Charité Universitätsmedizin Berlin, Berlin, Germany, ${ }^{7}$ Radiology, University of Alberta, Edmonton, Canada; ${ }^{8}$ Rheumatology, Copenhagen Center for Arthritis Research, Copenhagen, Denmark

Background: The diversity of MRI lesions in the sacroiliac joints of patients with axial spondyloarthritis (axSpA) has only recently been appreciated and consistent terminology, descriptions, and definitions have not yet been internationally accepted. The ASAS MRI group has generated updated consensus lesion definitions (ASAS_MRI_def ${ }^{\mathrm{n}}$ ) and these now require validation in multi-reader exercises to support widespread adoption for clinical practice and research.

Objectives: To assess the reliability of detection of active and structural lesions as defined by (ASAS MRI def ${ }^{n}$ ) on MRI images from the ASAS Classification Cohort (ASAS-CC) in a multireader ASAS exercise.

Methods: ASAS MRI def $^{n}$ were recorded in an eCRF that comprises global assessment (lesion present/absent) and detailed scoring (SPARCC SIJ inflammation, SPARCC SIJ structural). MRI images were available in a variety of formats (DICOM $(n=175)$, JPEG $(n=71)$, DICOM film( $(n=32))$ and sequences, axial and semicoronal orientations, from 278 of the 495 cases who had MRI performed in the ASAS-CC. Image quality was considered sufficient to record global data by 6 central readers in all cases. An additional reader assessed only images in DICOM format $(n=175)$. Detailed SPARCC scoring data was based only on assessment of images in DICOM format. Detection of lesions assessed as 